\title{
Auditory selectivity for the acoustic properties of conspecific mate-attracting signals in lower vertebrates and songbirds
}

This article was published in the following Dove Press journal:

Open Access Animal Physiology

25 May 2015

Number of times this article has been viewed

\section{H Carl Gerhardt}

Division of Biological Sciences, University of Missouri, Columbia, MO, USA
Correspondence: H Carl Gerhardt Division of Biological Sciences, University of Missouri, 2I 5 Tucker Hall, Columbia, MO 652II, USA

Email gerhardth@missouri.edu

\begin{abstract}
The interplay between conspecific senders and receivers both maintains the usual species specificity of acoustic communication and yet offers the potential for speciation provided that signals and preferences change in a parallel or coupled way. Acoustic signals commonly function in mate attraction and contribute to reproductive success. Such signals are especially prevalent in some lower vertebrates (some fish and many kinds of frogs) and birds in which prospective mates potentially assess variation in acoustic properties among signalers in order to choose an appropriate mate. Selectivity for specific ranges of values of different acoustic properties has been demonstrated by playback experiments, and neural correlates of this selectivity have been documented at various loci in the auditory system. Behavioral studies of temperature effects in lower vertebrates and learning in songbirds provide opportunities to predict short-term changes in neural activity. Demonstrating such "second-order" correlations increases our confidence that the selectivity of neurons or circuits for conspecific acoustic signals is not merely coincidental. With regard to parallel changes in signals and receiver selectivity over evolutionary time, this review describes the role of linkage disequilibria, the potential for genetic coupling between sender and receiver mechanisms, and how changes in cell numbers and size resulting from polyploidy may have facilitated multiple independent occurrences of "instantaneous" speciation.
\end{abstract}

Keywords: signals, acoustic communication, vertebrates, temperature, mates, frogs, fish

\section{Introduction}

The dominant function of acoustic communication in animals is mate acquisition. Individuals of one sex, usually males, produce signals that stimulate and attract a prospective mate. The goal of this review is to discuss examples of the physiological mechanisms and evolutionary processes that contribute to the selectivity of vertebrates with regard to specific properties of the signals used in mate acquisition, either directly or indirectly through territorial defense. The selectivity of female receivers with regard to natural variation in signals of their own species is usually more than sufficient to exclude attraction to the signals of other species. ${ }^{1}$ Nevertheless, a few welldocumented examples of reproductive character displacement - divergence in signals, female selectivity, or both in areas of sympatry between closely related taxa - indicate that communication systems can be affected by interactions between species. ${ }^{2}$ Here the adverse consequences of mating mistakes based on misidentification of signals constitute strong selection for species specificity of signals and especially of female selectivity. ${ }^{3}$ Regardless of the causes or interpretation of selectivity for conspecific acoustic signals, most of our insights about the underlying neural mechanisms come 
from studies that assess how neurons and neural circuits respond to conspecific signals as opposed to those of other, closely related species.

A prerequisite for robust studies of mechanisms and evolutionary process is to characterize the physical properties of mate-acquisition signals and how they vary within bouts of signaling and among males in a population. Behavioral studies can then identify the properties actually used in mate choice and their most effective values. This kind of information is usually summarized in a preference function, which plots the attractiveness of a series of acoustic stimuli as a function of the systematic variation of one of their acoustic properties. ${ }^{1}$ The next step is to assess the selectivity of the auditory system to different values of these same key stimuli. This review will describe examples of such studies in fish, ${ }^{4,5}$ frogs, ${ }^{6-12}$ and songbirds. ${ }^{13-19}$ I will highlight studies for which robust behavioral experiments have identified the key properties. Furthermore, studies demonstrating short-term, parallel changes in behavioral and neural selectivity arising from environmental factors, such as temperature in lower vertebrates and learning in songbirds, add to our confidence that auditory selectivity with regard to key acoustic properties is not merely coincidental.

While the match between specific signal properties and the selectivity of conspecific receivers is a form of "coupling" that is expected to promote stability of acoustic communication systems, the diversity of species with different acoustic signals testifies to the power of evolutionary change. Preference functions for key properties of acoustic signals can predict evolutionary changes within populations, and assessments of geographic variation in key signals and preferences can reveal the potential for speciation. Rapid speciation will, in general, depend on "coupling" mechanisms that promote parallel changes in the selectivity of receivers, and I will consider two such hypothetical processes, genetic coupling and parallel, "pleiotropic" effects of polyploidy.

\section{Information-bearing properties of acoustic signals}

One advantage of studying acoustic signals is that their analysis is relatively straightforward. ${ }^{1}$ The first step is to analyze samples of the same type of mate-attracting signal of numerous individuals in different populations. Properties that show relatively little variation within bouts of signaling in individuals (static properties as defined by Gerhardt ${ }^{20}$ ), between individuals within a population, and between all of the populations sampled are the most useful attributes for researchers for identifying the species of the signaler. ${ }^{1,20}$
Fine time-scale temporal properties usually fall into this category. These are short-duration $(<100 \mathrm{~ms})$ sounds with a consistent amplitude-time envelope, which, if organized into trains or trills, have a consistent pattern of repetition (Figure 1A; top trace). Sometimes, significant geographical differences in such properties do occur in wide-ranging species, however, and this divergence could represent the

A
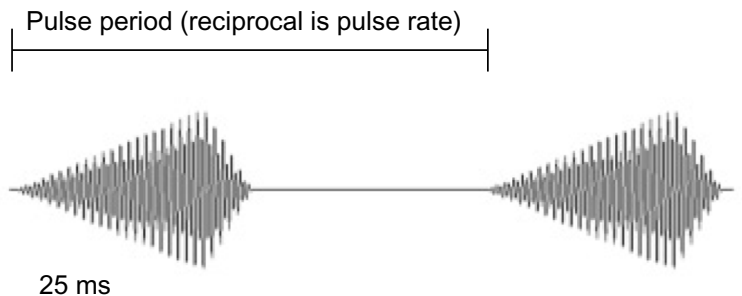

$25 \mathrm{~ms}$

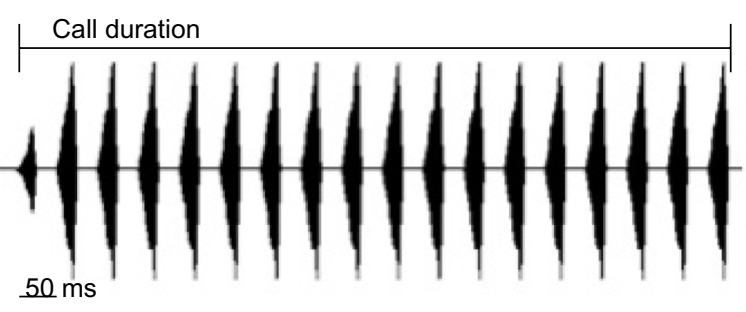

Call period (reciprocal is call rate)

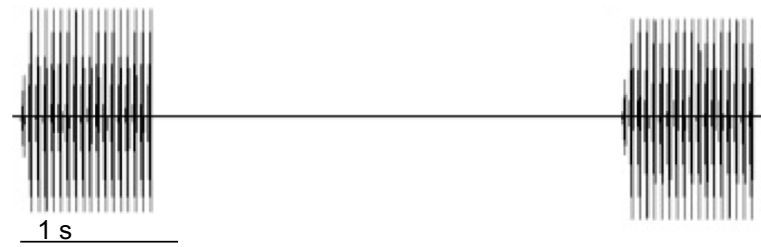

B

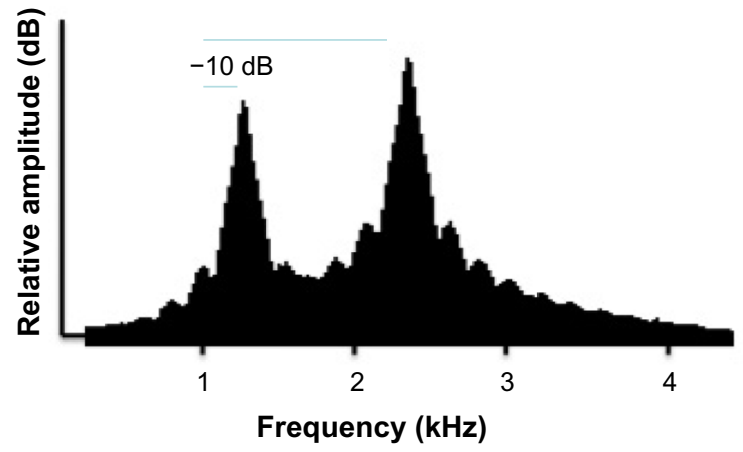

Figure I Examples of acoustic properties of mate-attracting signals.

Notes: (A) Oscillograms showing two sound pulses (top trace) within a call (middle trace). These are highly stereotyped (static) fine-scale temporal properties. The third trace shows two repetitions of the pulse train; call period and the number of pulses per call (call duration) are highly dynamic properties, ${ }^{20}$ (B) frequency spectrum - energy averaged over one call. The signals illustrated here are synthetic, computer-generated signals modeled after a typical call and using values close to the mean for a representative population of gray tree frogs (Hyla versicolor). Playbacks of such a synthetic call attracted gravid females as effectively as did playbacks of prerecorded natural calls. ${ }^{44}$ Reproduced with permission from John Wiley and Sons. Experimental evidence of multivariate female choice in gray treefrogs (Hyla versicolor): evidence for directional and stabilizing selection. Gerhardt HC, Brooks R. Evolution. () 2009 Gerhardt and Brooks. Journal compilation (C) 2009 The Society for the Study of Evolution. ${ }^{28}$ 
early stages of speciation. ${ }^{7,20,21}$ Other static properties, such as the spectral content (eg, dominant components and their frequencies, frequency modulation) of the signal (Figure 2B), typically vary significantly among males within a population and often correlate with a signaler's size and weight, thus potentially conveying information about these attributes to prospective mates. ${ }^{1,22,23}$ The greatest progress in understanding neural mechanisms underlying selective responses to conspecific calls, especially in lower vertebrates, comes from studies assessing the behavioral relevance and selectivity of the auditory system with regard to static acoustic properties. ${ }^{1}$

Other acoustic properties may vary significantly within a bout of calling by an individual (Figure 1A, bottom trace; Figure 2). ${ }^{1,20,24,25}$ High values of such dynamic properties may indicate a male's physical condition because of the prevalence of positive correlations - at least in lower vertebrates - with energetic costs. ${ }^{1,24,25}$ Despite the variability within individual bouts of calling, the variability of dynamic properties may still differ significantly among males in a population, thus

\section{A}

\begin{tabular}{|c|c|c|c|c|c|}
\hline & $\begin{array}{l}\text { Dominant } \\
\text { frequency }\end{array}$ & $\begin{array}{l}\text { Pulse } \\
\text { rate }\end{array}$ & $\begin{array}{c}\text { Call } \\
\text { duration }\end{array}$ & $\begin{array}{l}\text { Pulse } \\
\text { number }\end{array}$ & $\begin{array}{l}\text { Call } \\
\text { rate }\end{array}$ \\
\hline H. versicolor & 0.8 & 1.5 & 16.4 & 15.0 & 25.4 \\
\hline Н. cinerea & 0.5 & & 3.7 & & 12.3 \\
\hline . crucifer & 0.8 & & 4.0 & & 16.0 \\
\hline
\end{tabular}

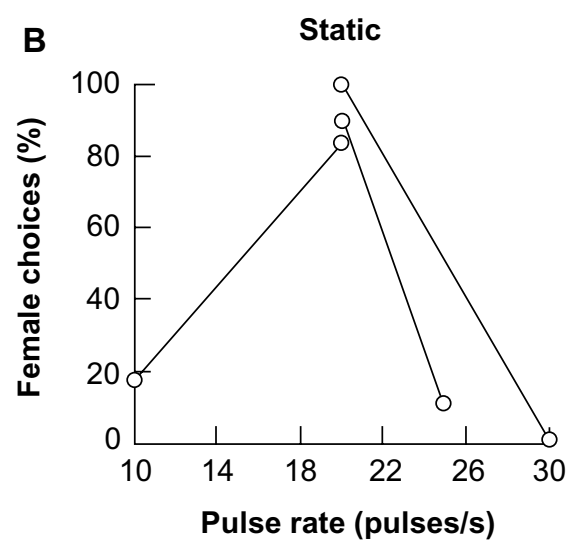

Dynamic
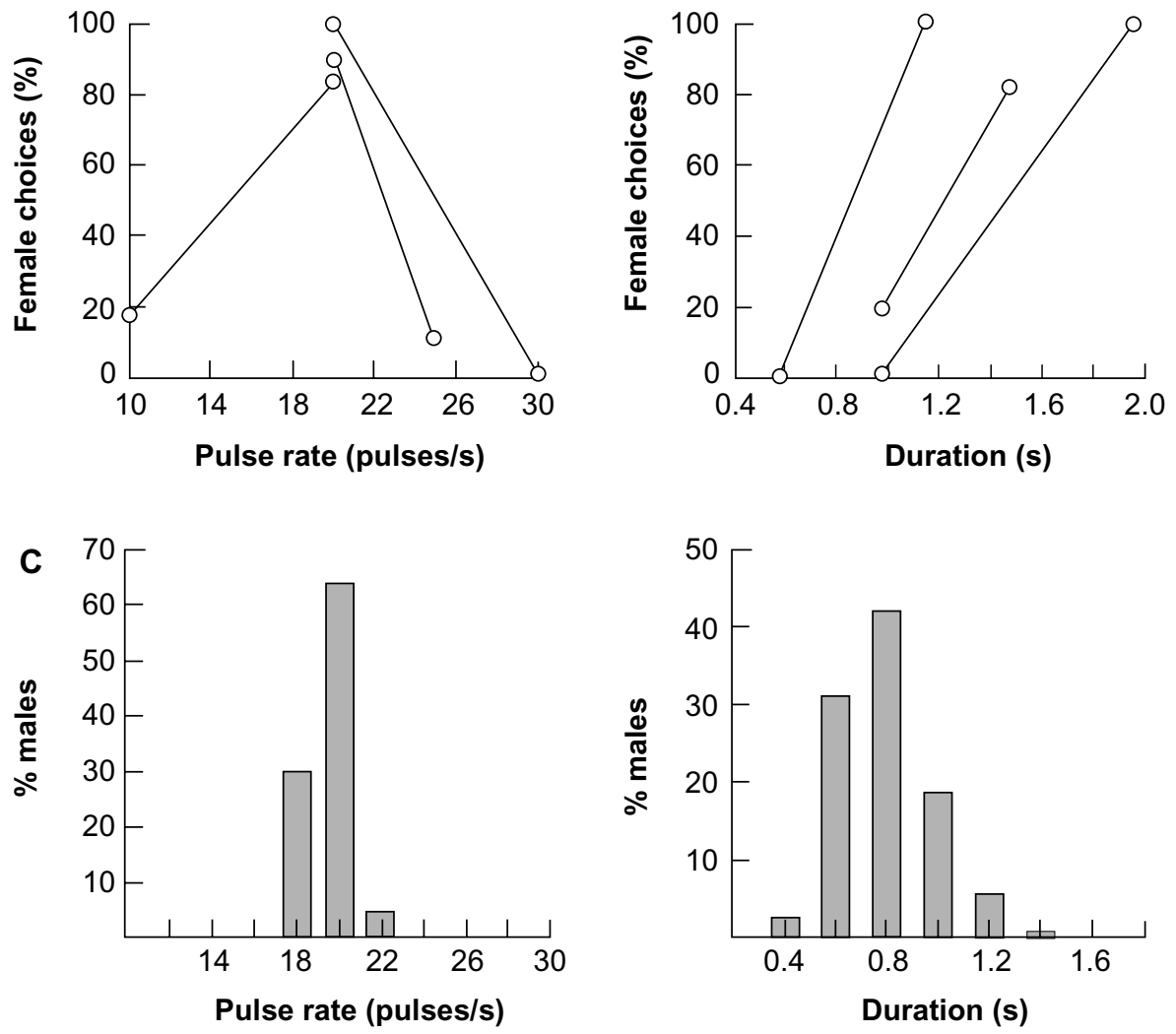

Figure 2 Static and dynamic acoustic properties and typical patterns of selection.

Notes: (A) Table showing the average values of the coefficient of variation (standard deviation as a percentage of the mean) within bouts (series of 5-30 calls) of males of three kinds of tree frogs (Hyla versicolor is eastern gray tree frog; Hyla cinerea is green tree frog; Pseudacris crucifer is spring peeper). Values in unshaded boxes are representative of static properties; values in shaded boxes are representative of dynamic properties (see text and Gerhardt $\mathrm{HC}^{20}$ ). (B) Results of two-alternative forced-choice tests with females of $\mathrm{H}$. versicolor. Each line connects points showing the proportions of females choosing one of the alternatives. For example, in the left panel all females tested (I00\%) chose an alternative with a pulse rate of 20 pulses/s when the alternative had a pulse rate of 30 pulses $/ \mathrm{s}$ ( $0 \%$ in the graph). (C) Distributions of values of pulse rate (left) and call duration (right) in populations from which females were collected. The pattern of preference for pulse rate (a static property) is stabilizing, whereas the pattern of preference for call duration (a dynamic property) is highly directional. This article was published in Encyclopedia of Neuroscience, 3rd ed, Squire LR, Copyright Elsevier 2004. ${ }^{66}$ 
allowing prospective mates to use such properties to assess these individuals. ${ }^{24}$ In the eastern gray tree frog (Hyla versicolor), for example, call duration (number of pulses in the advertisement trill) is heritable in a multivariate context. ${ }^{26}$ That is, despite variation in this and other behaviorally relevant acoustic properties, selection on variation in call duration has the potential to cause an evolutionary change in this property. Furthermore, in an experimental analysis of laboratory-generated half-sibs, offspring of fathers with long-duration calls, which are energetically costly to produce, had higher growth rates as tadpoles and juveniles than did offspring of fathers with short-duration calls. ${ }^{27}$ On the one hand, preferences based on differences in dynamic properties are usually weaker than preferences based on differences in pulse rate in terms of their intensity dependence. That is, reducing the playback level of a preferred alternative by just 3-6 dB relative to that of an alternative with a different value of a dynamic property such as call duration or call rate usually abolishes or even reverses the preference. ${ }^{24} \mathrm{On}$ the other hand, preferences based on dynamic properties are often highly directional, in that even values outside the range of variation in male calls in the same population may be preferred to average values (Figure 2B and C), perhaps because, as stated earlier, high values may be indicative of a superior mate. ${ }^{24,25,27}$ We know relatively little about the neural mechanisms underlying such preferences, but high values of these properties should result in stronger responses in auditory neurons up to the point of sensory adaptation. Preferences based on static properties are generally stabilizing, in that females prefer signals with values at or near the mean in the population.

Most signals are composed of multiple static and dynamic properties. Preferences based on multiple properties will of course interact, and some will be more heavily weighed than others. In gray tree frogs, a behavioral study of multivariate selection, nevertheless, found that the stabilizing preferences for static properties and directional preferences for dynamic properties were maintained when the values of both kinds of properties were varied simultaneously and systematically over their natural ranges of variation. ${ }^{28}$ The relative importance of static and dynamic properties can also differ between populations. Pulse rate, for example, was more important than call duration in populations of Cope's gray tree frog (Hyla chrysoscelis) in which its genetically incompatible sibling, the eastern gray tree frog $(H$. versicolor), was present (sympatry) than in populations in which $H$. versicolor was absent (allopatry). ${ }^{3}$ Pulse rate and pulse duration, but not call duration, differ between the two species. Mistakes based on a failure to mate with a male producing calls with conspecific values of pulse rate are much more serious (production of nonviable or sterile offspring) than mistakes based on call duration (perhaps the production of somewhat less fit offspring). Hence in areas where both species occur, females are constrained to choose mates mainly on the basis of pulse rate.

In higher vertebrates, another layer of complexity is introduced by learning. Here individuals may not only learn the signals of social partners and rivals but signal properties that characterize the region where they occur, ie, dialects. ${ }^{29}$ The signal properties that mediate these recognition tasks are likely to change somewhat during the lifetime of individuals, and gene flow between populations with different dialects can alter signal structure over longer time periods.

\section{Auditory selectivity for species- typical acoustic properties}

Just because some acoustic property is species-specific does not mean that females use this property in evaluating a prospective mate. Behavioral experiments using synthetic signals have shown that even stereotyped properties that occur in the signals of every individual of a given species may be irrelevant for signal recognition. For example, female spring peepers (Pseudacris crucifer) and gray tree frogs do not show a preference between synthetic advertisement calls that are frequency modulated to the same extent as in natural calls and synthetic calls lacking frequency modulation altogether (Figure 3). 1,30-32 Whether these animals cannot perceive the difference or simply ignore the difference is an open question. In any event, studies of the selectivity of the auditory sys-

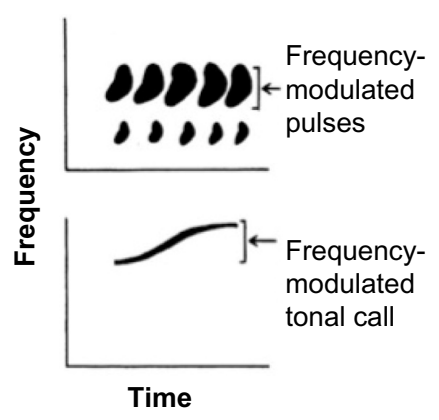

Figure 3 Cartoons of sonograms (frequency versus time) of part of the advertisement trill of a gray tree frog (Hyla versicolor) (top trace) and one advertisement call of a spring peeper (Pseudacris crucifer) (bottom trace).

Notes: Even though the frequency changes by $300-500 \mathrm{~Hz}$ from beginning to end in the pulses and call, females did not show a preference for synthetic calls with such frequency modulation to alternatives in which frequency was held constant. ${ }^{35,46}$ Copyright (C) 988. John Wiley \& Sons Ltd. Reproduced from Gerhardt, HC. Acoustic properties used in call recognition by frogs and toads. In Fritzsch B, Ryan MJ, Wilczynski W, Hetherington TE, Walkowiak W eds. The Evolution of the Amphibian Auditory System. John Wiley and Sons, NY, USA. 455-483. ${ }^{67}$ 
tem with regard to species-typical properties of conspecific signals are far more valuable when behavioral studies have already established the relevance of those properties. Indeed, studies of auditory mechanisms are also more powerful if they employ such synthetic signals as stimuli rather than recordings of complex calls or, at the other extreme, simple tones and clicks. ${ }^{1,33}$

\section{Auditory selectivity in lower vertebrates: spectral properties}

Birds and mammals usually have a broad and sensitive hearing range that encompasses the frequencies of the signals of their own and other species, whereas lower vertebrates such as fish, frogs, and toads typically have limited and even discontinuous hearing ranges and generally higher auditory thresholds. ${ }^{1,46,33}$ These animals have served as particularly tractable models for acoustic communication because in many species mate choice is based solely on the acoustic modality, and reproductively primed females readily respond to playbacks of prerecorded and synthetic calls, whose properties can be systematically varied. ${ }^{1,33}$ By contrast, many vertebrate groups - especially birds and mammals - use other modalities in addition to acoustic signals to choose mating partners. The recognition of mating partners based on multimodal input is probably the reason that there is no robust example of reproductive character displacement in birds or mammals. ${ }^{2}$ The same hypothesis may apply to auditory selectivity. The poor classification performance of multisensory neurons in prefrontal cortex of macaques in response to auditory stimuli, for example, was attributed to the lack of simultaneous visual (facial) input. ${ }^{34}$

The discontinuity in the hearing range of frogs and toads arises because these animals have two different auditory inner ear organs, each tuned to a different frequency range. The more complex amphibian papilla is tuned to relatively low-frequency sounds, and the basilar papilla, to relatively high-frequency sounds. ${ }^{1,33}$ If there is one emphasized frequency or frequency band in the mate-attracting signals of a species, it usually corresponds at least approximately to the frequency selectivity of one of these organs, and if there are two such bands, both organs are likely to be effectively stimulated (Figure 4). ${ }^{6}$ Tuning of this nature is also observed at the level of different populations of the same species in which call frequency and peripheral auditory tuning are similar., ${ }^{7,33}$ These so-called matched-filters presumably help animals in mixed-species choruses deal with the "noise" produced by the vocalizations of other frogs and insects and perhaps some forms of environmental noise. I emphasize, however, that

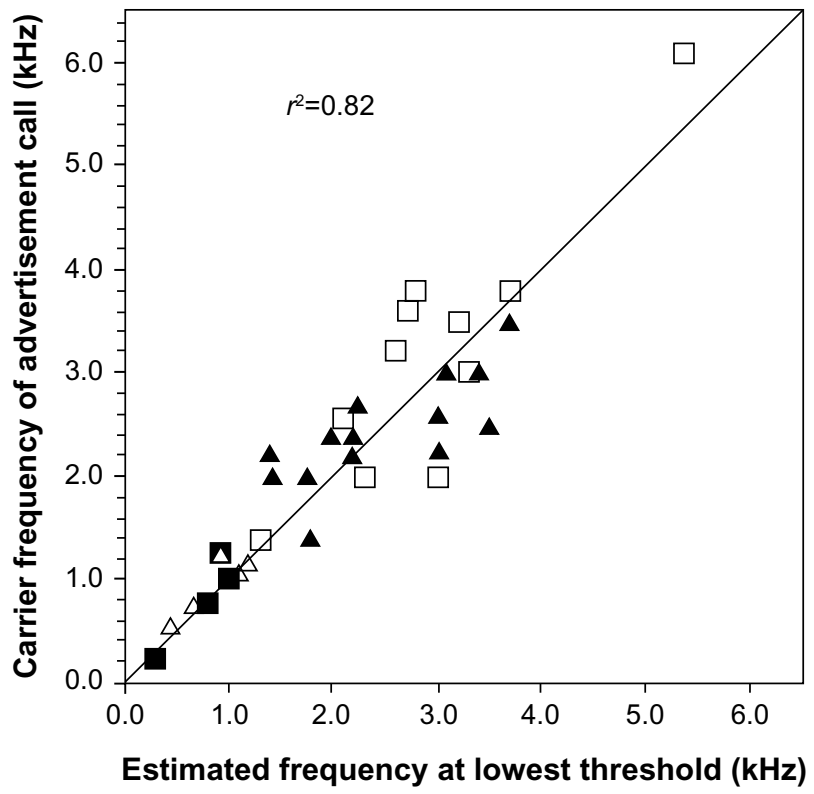

Figure 4 Correlation between spectral (frequency) peaks in the mate-attracting calls of species of frogs and toads and estimates of the minimum auditory thresholds of the same species.

Notes: These estimates were obtained from single-unit (auditory nerve) and multiunit spike data and evoked potentials from the midbrain (torus semicircularis). Solid symbols: closed-system auditory stimulation; open symbols: free-field stimulation; squares: low-frequency sensitivity attributed to the amphibian papilla; triangles: high-frequency sensitivity attributed to the basilar papilla. The line shows where points would lie if the correlation were perfect. Adapted from Gerhardt HC, Schwartz JJ. Auditory tuning and frequency preferences in anurans. In: Ryan MJ, editor. Anuran Communication. Washington, DC: Smithsonian Press; 200 I:73-85. ${ }^{6}$

tuning by both organs, but especially the basilar papilla, is relatively weak, and the auditory system and the whole animal itself respond reliably to frequencies well outside the band to which these organs are tuned, especially when acoustic stimuli are presented at high amplitudes. ${ }^{1,22}$ Moreover, the most potent source of masking interference will be the signals of other males of the same species, which will of course have very similar spectral properties. Such masking is a serious problem in large choruses of species with long or rapidly repeated signals, ${ }^{35}$ and there is a large literature concerning how individuals ameliorate this problem. ${ }^{36-38}$ Extreme examples of the filtering of environmental noise are frogs that produce signals with ultrasonic frequencies to which their basilar papillae are sensitive. These species communicate along fast-flowing streams, where the sounds of flowing water generate intense low-frequency noise. ${ }^{39}$

\section{Auditory selectivity in lower vertebrates: fine-scale temporal properties}

The fine-scale temporal properties of the acoustic signals of fish and frog signals are usually static and species-specific, and their repertoire usually contains at least one kind of signal that consists of trains of clicks or pulses (Figure 1A). ${ }^{1,4}$ 
Behavioral studies often have found that females are highly selective with regard to the duration, rise-fall characteristics, and repetition rate of these elements. ${ }^{1,4,9,31,33,40-42}$ At the peripheral level, auditory neurons usually copy or synchronize firing to discrete temporal elements such as pulses or even to a particular phase of a sinusoidal signal up to repetition rates or frequencies where phase-locking breaks down. ${ }^{1,4}$ The degree of synchronization is often equivalent over a broad range up to this point and often includes repetition rates typical of the pulsed signals of other species. The burden of selectivity for different values of fine-scale properties (duration, rise-fall characteristics, rate, frequency) thus falls on circuits in higher auditory centers, primarily in the midbrain, in particular in the torus semicircularis (analog of the inferior colliculus in higher vertebrates) (Figure 5A)..$^{1,4,12,33}$ In lower vertebrates there is an additional complication because temperature affects the values of these fine-scale properties. Hence without some compensation in the receiver, which I discuss later, the selectivity for conspecific calls could be compromised at some temperatures.

Bass and McKibben ${ }^{4}$ provide an extensive review of the processing of natural acoustic signals in the midshipman (Porichthys notatus) and other fish. Male midshipman and their toadfish relatives produce long-lasting hums with little variation in frequency. These low-frequency signals are produced by territorial males and attract gravid females (Figure 6); pure-tone signals are also effective in this regard. Auditory neurons in the eighth nerve show broad tuning to the frequencies in conspecific hums but also strong, sustained synchronization to individual and two-component signals consisting of the fundamental and second harmonic (around 100 and $200 \mathrm{~Hz}$, respectively). These tonal signals are thus processed in both the frequency and time domains to some extent. During territorial establishment, males also produce trains of grunts (Figure 6), which serve to repel rivals and which do not attract females. Experiments using two-component
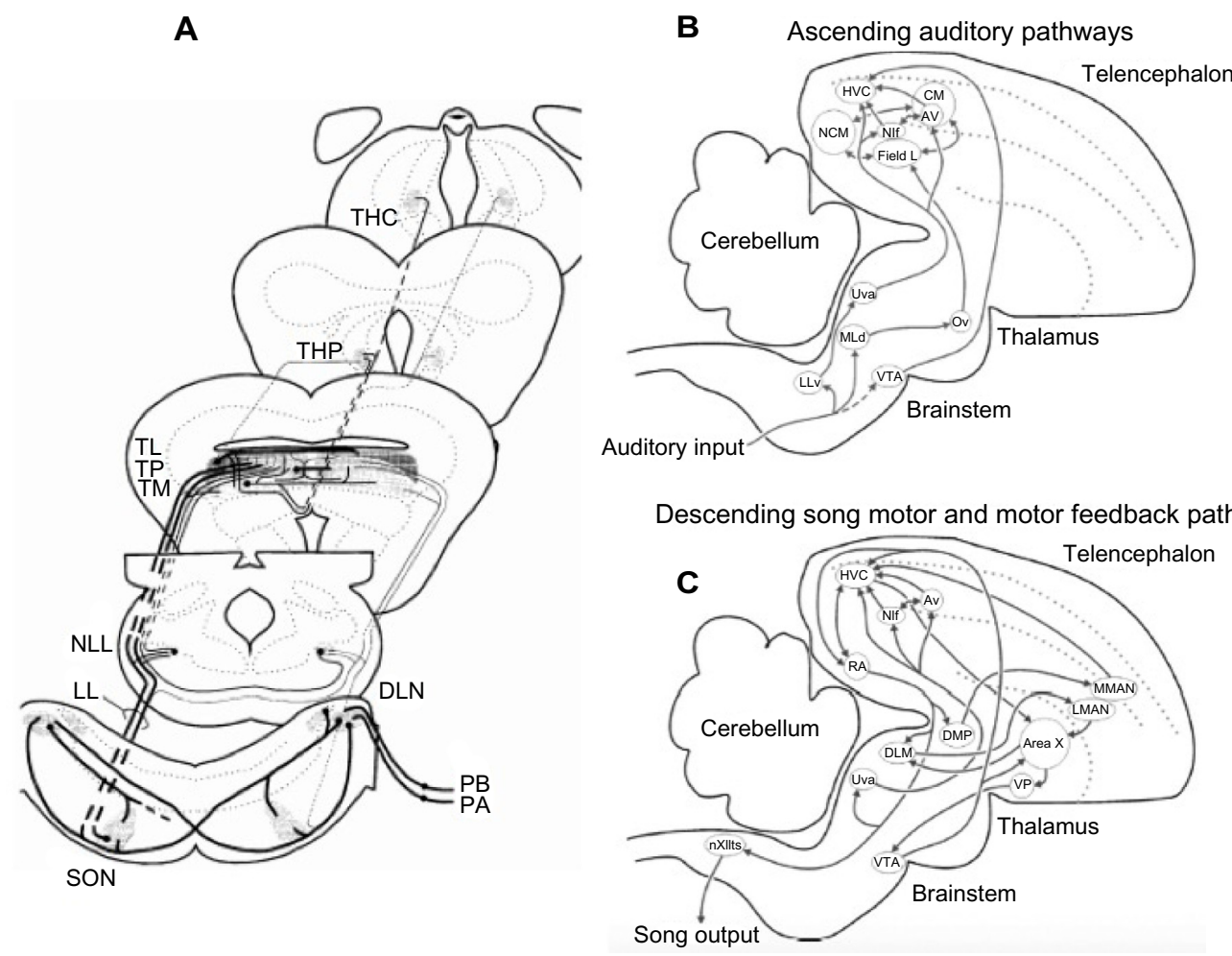

Figure 5 Major ascending auditory pathways in frogs and birds.

Notes: (A) Auditory pathway in anurans. Very little information is available about auditory selectivity in the thalamus, where refractory periods are extremely long; lesions of the torus semicircularis abolish phonotaxis, but selective phonotaxis occurs after extensive lesions of the thalamus. ${ }^{49}$ (B) Ascending auditory system of a songbird. (C) Descending song motor and feedback pathway. HVC is the major sensory-motor center. Details are provided in Prather ${ }^{18}$ and Jarvis. ${ }^{50}$ From Gerhardt HC, Huber F. Acoustic Communication in Insects and Anurans: Common Problems and Diverse Solutions. Chicago, IL: University of Chicago Press; Copyright @ 2002.' and adapted from Hearing Res. Volume 305. Prather JF. Auditory signal processing in communication: perception and performance of vocal signals, Pages 144-155. Copyright @ 2013 with permission from Elsevier. ${ }^{18}$

Abbreviations: PB, auditory nerve fibers from basilar papilla; PA, auditory nerve fibers from amphibian papilla; SON, superior olivary nucleus; DLM, dorsolateral nucleus; LL, lateral lemniscus; NLL, nucleus of the lateral lemniscus; TL, laminar nucleus of the torus semicircularis (inferior colliculus); TP, principal nucleus of the torus; TM, magnocellular nucleus of the torus; THP, posterior nucleus of the thalamus; THC, central nucleus of the thalamus; LLv, ventral nucleus of the lateral lemniscus; VTA, ventral tegmental area; MLd, nucleus mesencephalicus lateralis; Ov, nucleus ovoidalis; Uva, nucleus uvaeformis; Field L, avian primary auditory cortex; NCM, caudomedial nidopallium; NIf, nucleus interface of the nidopallium; Av, nucleus avalanche; CM, caudal mesopallium; HVC, hypostriatum ventral pars caudale (high vocal center); Area X, specialized region of the avian striatum; DLM, dorsolateral nucleus of the thalamus; DMP, nucleus dorsomedialis posterior thalami; LMAN, lateral magnocellular nucleus of the anterior nidopallium; MMAN, medial magnocellular nucleus of the anterior nidopallium; RA, robust nucleus of the arcopallium; VP, ventral pallidum; $\mathrm{nXIlts,} \mathrm{hypoglossal} \mathrm{nucleus,} \mathrm{tracheosyringeal} \mathrm{nerve} \mathrm{(} 2$ th cranial nerve nucleus); CM, an area where neurons are highly selective for conspecific song. 
Midshipman sounds
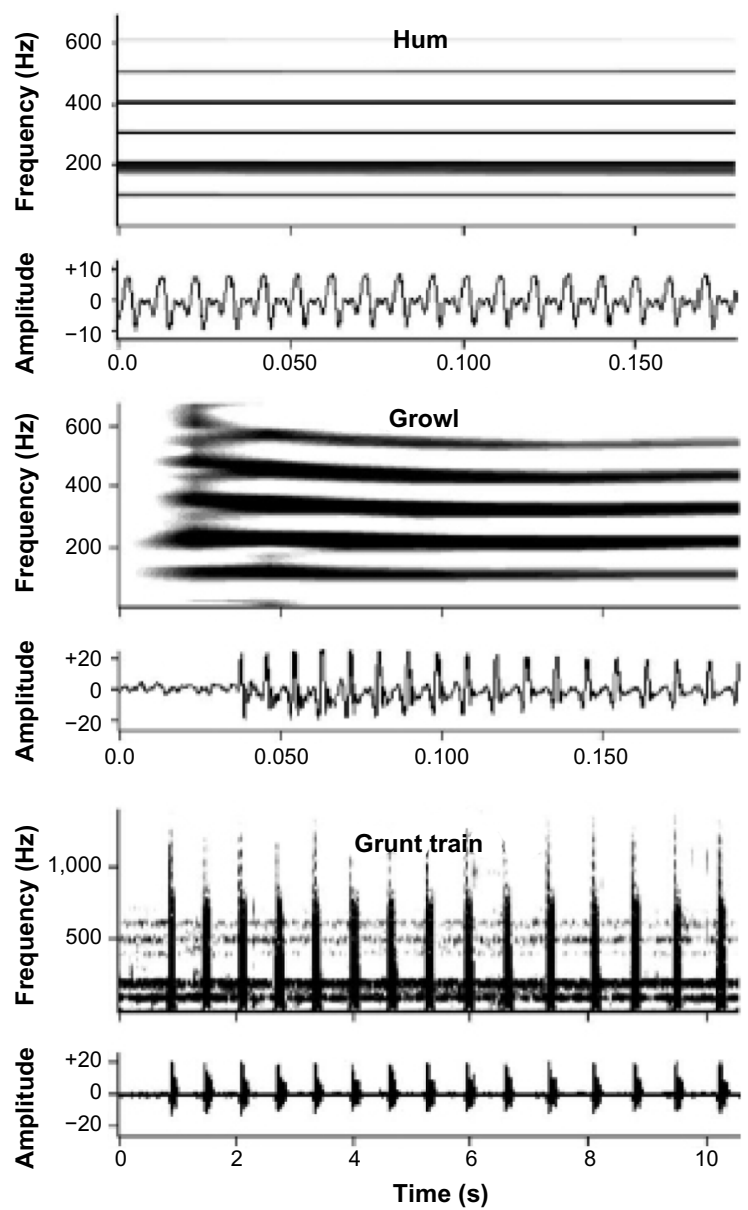

Figure 6 Sonograms (top) and oscillograms (bottom) of the acoustic signals of plainfin midshipman fish (Porichthys notatus).

Notes: Shown are representative excerpts from a I min $48 \mathrm{~s}$ hum, a $4.64 \mathrm{~s}$ growl, and 15 of 22 grunts in a growl train that were recorded from a male on his nest. Reprinted from Progress in Neurobiol, Volume 69, Bass AH, McKibben JR. Neural mechanisms and behaviors for acoustic communication in teleost fish, Pages I-26. Copyright (C) 2003, with permission from Elsevier. ${ }^{4}$ ("beat") stimuli, which mimic the sounds that occur when two males with different hum frequencies are nearby, showed that synchronization to the envelope of the beats occurred in the firing pattern of eighth nerve fibers (Figure 7). As the beat frequency increased, these cells showed a bursting pattern similar to that elicited by trains of grunts. The difference in firing pattern at the eighth nerve - sustained for long tonal stimuli and synchronized to the pattern of amplitude modulation in more complex signals - is likely to be the basis for discrimination of these two signals in the midshipman at higher levels in the auditory system. Midbrain auditory neurons showed frequency selectivity similar to that of auditory nerve fibers but differed in the temporal properties that resulted in synchronization to beats and were more likely to show adaptation to long-duration signals. ${ }^{4}$

In frogs, neurophysiological studies have described many auditory neurons in the midbrain that are selective for values of fine-scale temporal properties of known relevance in mate recognition. In leopard and Pacific chorus frogs, for example, one class of neurons is highly selective for pulserepetition rate (or intervals); these cells have the additional response property of requiring some minimum number of correct intervals before beginning to fire; still other neurons are selective for pulse duration. ${ }^{9-12}$

\section{A "second-order" correlation between behavioral and auditory selectivity}

Thus far I have discussed correlations between behavioral selectivity for acoustic properties and seemingly similar selectivity in auditory neurons or other neural responses. But
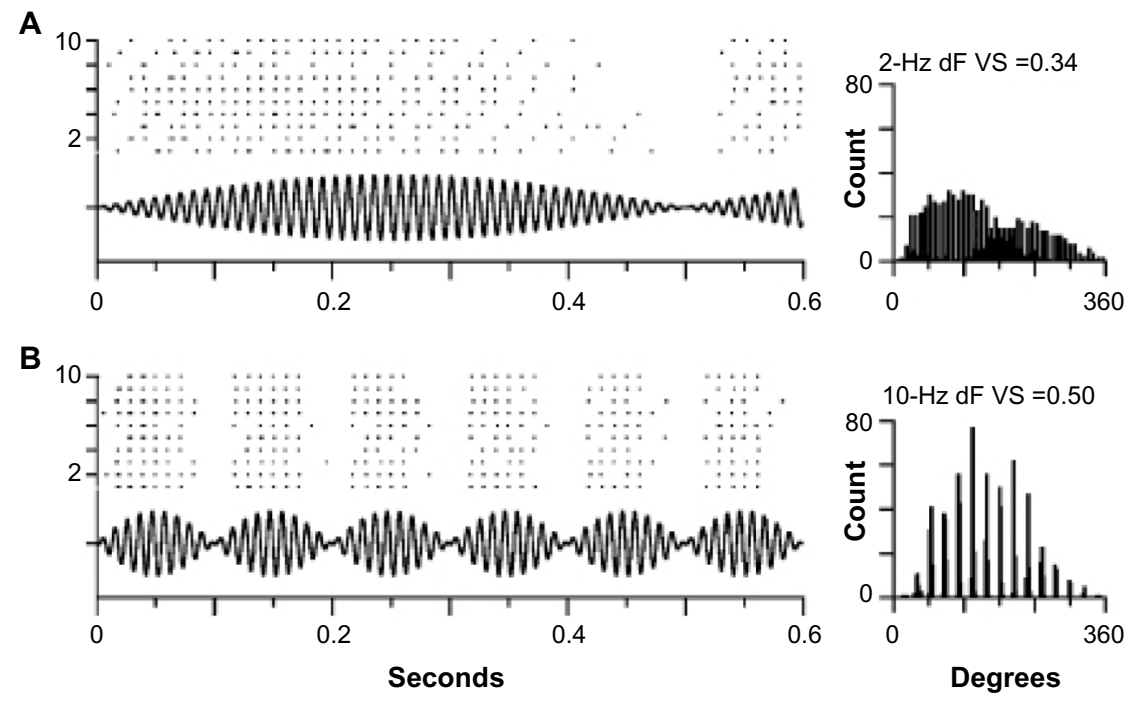

Figure 7 Encoding of the temporal structure of beats (oscillograms) with different beat frequencies.

Notes: Raster plots of spike times from recordings of the auditory nerve are shown above each oscillogram, and beat period histograms are to the right. (A) Stimulus with a beat frequency of $2 \mathrm{~Hz}$; (B) Stimulus with a beat frequency of $10 \mathrm{~Hz}$. Notice how the pattern of spikes is synchronized with and encodes the beat frequency. Reprinted from Progress in Neurobiol, Volume 69, Bass AH, McKibben JR. Neural mechanisms and behaviors for acoustic communication in teleost fish, Pages I-26. Copyright $@$ 2003, with permission from Elsevier. ${ }^{4}$ 
these are just correlations, and very many neurons at all levels of the auditory system show little, if any, such selectivity. Tones and noise bursts may drive them just as effectively as or better than species-typical signals. The question then arises, is there any further evidence that auditory neurons that are selective for species-typical properties really do function in mate identification? Or is this selectivity merely coincidental in the sense that some selectivity would be expected merely by chance in a large sample of neurons?

As discussed earlier, the fine-scale temporal properties of the signals of lower vertebrates are often highly temperature-dependent. The correlation between temperature and pulse rate of the advertisement calls of gray tree frogs, for example, is well above 0.9 so that in $H$. versicolor there is a rate increase of about 1 pulse $/ \mathrm{s}^{2} /{ }^{\circ} \mathrm{C}$ and in $H$. chrysoscelis the rate increase is more than 2 pulses $/ \mathrm{s}^{2} /{ }^{\circ} \mathrm{C}$ (Figure $\left.8 \mathrm{~A}\right) .{ }^{43}$ These are biologically significant effects because male frogs call and
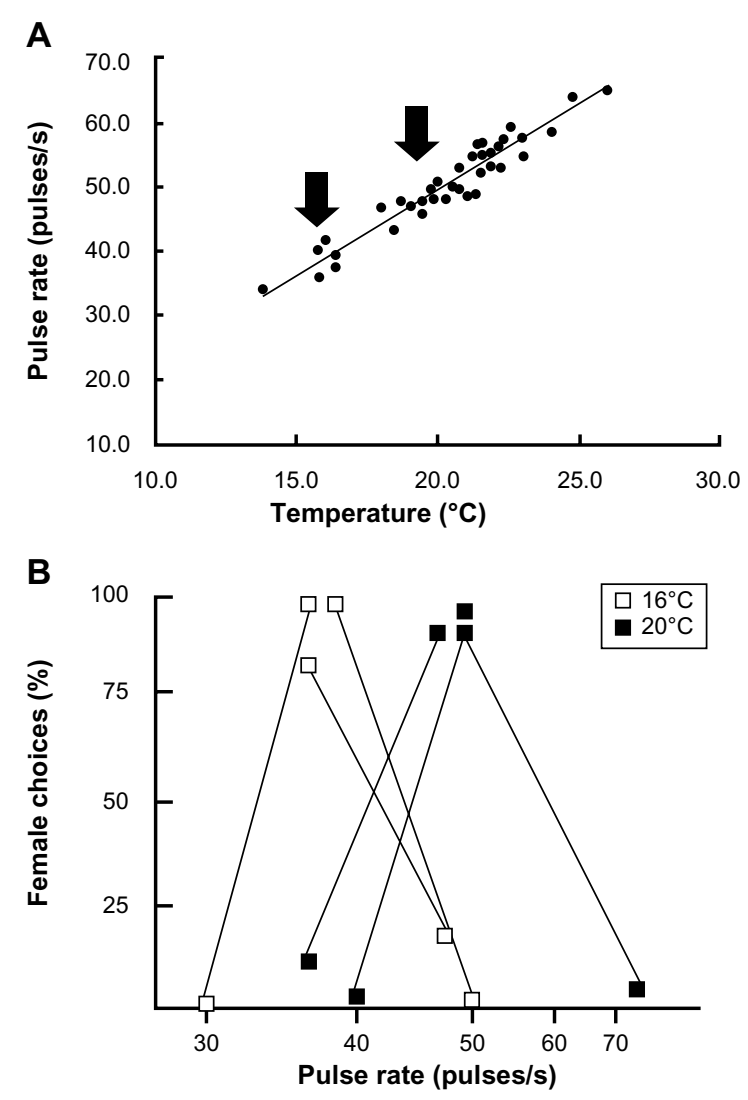

Figure 8 Temperature coupling in Cope's gray tree frog (Hyla chrysoscelis). Notes: (A) Scatter diagram and fit line for the regression of pulse rate on body temperature from a sample of males in central Missouri. The $r$ value from the relationship was 2.39. (B) Results of two-stimulus, forced-choice playback experiments with synthetic calls with pulse rates typical of males calling at $16^{\circ} \mathrm{C}$ and $20^{\circ} \mathrm{C}$, respectively (see arrows in part $\mathbf{A}$ ). Lines connecting open squares show the proportions of females tested at $16^{\circ} \mathrm{C}$ choosing an alternative of 40 pulses/s to alternatives with lower or higher values; lines connecting closed squares show the proportions of females tested at $20^{\circ} \mathrm{C}$ choosing an alternative of 50 pulses $/ \mathrm{s}$ to alternatives of lower or higher values. breed over a range of temperature from about $15^{\circ} \mathrm{C}-30^{\circ} \mathrm{C}$. Females show discrimination between signals, with differences in pulse rate corresponding to those observed between the calls of males with body temperature differences of just $4^{\circ} \mathrm{C}-5^{\circ} \mathrm{C}$. Because females prefer pulse rates that correspond to those produced by a male calling at about the same temperature at which they are tested, this match has been termed temperature coupling (see Figure $8 \mathrm{~B}$ for an example). ${ }^{32,44}$ Temperature coupling also occurs in midshipman fish ${ }^{45}$ and acoustic insects. ${ }^{1}$ Studies of auditory neurons in the midbrain of eastern gray tree frogs (H. versicolor) showed that some cells that are selective for pulse rate show similar shifts, both in terms of populations and even individual neurons (Figure 9). ${ }^{8}$ This interpretation has to be qualified somewhat because more recent experiments showed that recognition in $H$. versicolor actually depends largely on pulse duration. ${ }^{42}$ Nevertheless, because pulse duration, like pulse rate, is also temperature-dependent, ${ }^{43}$ midbrain neurons selective for this property must be responsible for temperature coupling in this species, whereas something like bandpass neurons selective for pulse rate alone would suffice in H. chrysoscelis. ${ }^{42}$

This kind of second-order correlation is also illustrated by a counterexample in which temperature changes abolish a tuning match between neural selectivity in the central nervous system and the frequency of some components in conspecific calls. In green tree frogs (Hyla cinerea), the emphasized frequencies of their noisy calls are affected very little by changes in temperature, but female preferences based on the frequency of the low-frequency spectral peak

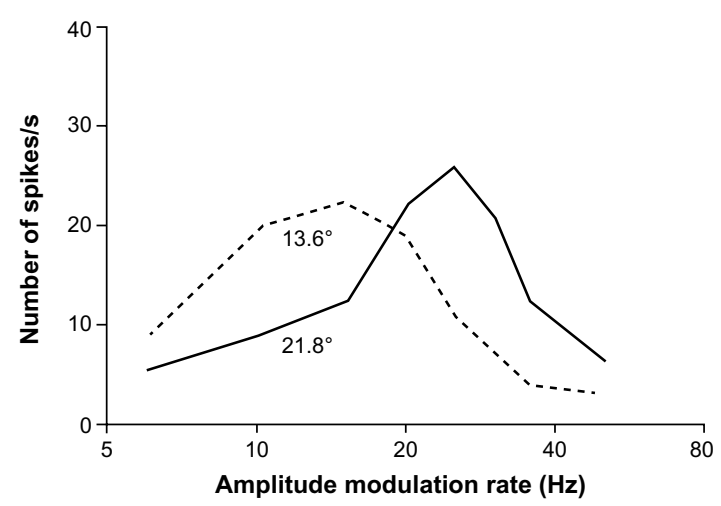

Figure 9 Temperature dependence of a temporally selective neuron in the midbrain of the eastern gray tree frog (Hyla versicolor).

Notes: The bandpass "tuning" of the neuron shifted with temperature ${ }^{8}$ as did the pulse-rate preferences of female frogs tested at different temperatures. ${ }^{44} \mathrm{~A}$ more recent study ${ }^{42}$ showed that females of this species use pulse duration rather than pulse rate to identify conspecific advertisement calls, but pulse duration is also temperature-dependent to nearly the same extent as pulse rate. ${ }^{45}$ Reprinted from Brain Res, Volume 359, Brenowitz EA, Rose G, Capranica RR. Neural correlates of temperature coupling in the vocal communication system of the gray treefrog (Hyla versicolor), Pages 364-367. Copyright (C) 1985, with permission from Elsevier. ${ }^{8}$ 

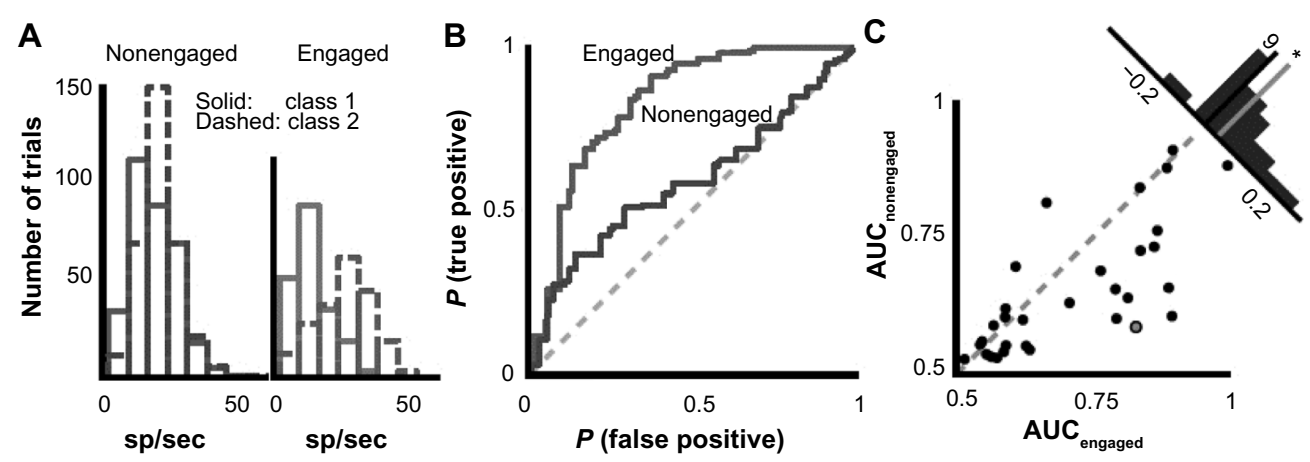

Figure 10 Increase in selectivity of a representative auditory neuron in the forebrain (caudal mesopallium) of a songbird (starling) when engaged in a vocal recognition task whose properties were controlled by the investigators.

Notes: (A) Histogram of firing rates in nonengaged and engaged states to different classes of stimuli in terms of how the bird was conditioned (GO or NO_GO to stimulus). (B) Receiver operating curve; the greater area under the curve in the engaged condition versus the nonengaged condition indicates greater discriminability of signals in the engaged condition. (C) Areas under the curve in panel $\mathbf{B}$ for all neurons recorded in the study that showed significant task discrimination in both conditions; the distribution of differences (top right histogram) shows neurons recorded in the engaged condition were more selective at the population level. The gray-centered point shows where the representative neuron (panels A and B) falls in the distribution. *Indicates statistically significant difference. From Knudsen DP, Gentner TQ. Active recognition enhances the representation of behaviorally relevant information in single auditory forebrain neurons. Journal of Neurophysiology. 2013;109:1690-I703.14

to which the amphibian papilla is tuned shift to values much lower than those found in the calls of conspecific males at low temperatures. ${ }^{46,47}$ Short-term changes in frequency preferences based on the frequency of the high-frequency peaks, to which the basilar papilla is most sensitive, do not change with temperature. ${ }^{47}$ Tuning in the amphibian papilla depends on temperature-dependent, metabolically active processes, whereas the basilar papilla functions basically as a resonant system. ${ }^{48}$ In this example, the counterintuitive behavioral experiments were suggested by neurophysiological results at temperatures well below the range at which green tree frogs breed. ${ }^{47}$ The preferences predicted at those temperatures, which could promote mismatings with a closely related species, are rarely if ever expressed in nature. ${ }^{47}$

\section{Auditory selectivity in songbirds: secondary correlations}

In contrast to fish and amphibians, birds and mammals can discriminate between signals that differ only slightly in frequency in a virtually intensity-independent fashion. ${ }^{15}$ Neurons selective for the same kinds of species-identifying acoustic properties discussed earlier for lower vertebrates have been described, as well as selectivity for signals of specific individuals and even the bird's own songs. ${ }^{13-19}$ Another distinction from lower vertebrates is the prominence of auditory processing in higher brain nuclei such as the thalamus, auditory cortex, and homologs of the auditory cortex such as the caudal mesopallium and associated structures in songbirds (Figure 5B). ${ }^{13-16}$ Although neural processing of communication and other signals also occurs at the level of the midbrain in these and other higher vertebrates, ${ }^{17-19}$ the role of auditory processing in the thalamus and higher centers in frogs is likely to be inconsequential in the context of mate choice. Evoked auditory responses in the thalamus of green tree frogs have exceedingly long refractory periods, ${ }^{47}$ and extensive lesions to the thalamus did not disrupt selective phonotaxis in gray tree frogs. ${ }^{49}$

Learning plays a major role in signal production as well as recognition in some avian groups such as songbirds. ${ }^{13-16,21}$ Many important insights about vocal-auditory coordination have come from neurophysiological studies of awake and behaving animals producing song ${ }^{18}$ or engaged in learning and discrimination tasks. ${ }^{13-15}$ I suggest that changes in neural activity observed during these behaviors represent another opportunity to explore a set of secondary correlations that increase our confidence that some of the neurons and circuits responding selectively to conspecific signals are in fact major contributors to recognition processes occurring during mate acquisition or the defense of territories. Changes in neural activity associated with learning of species-specific vocalizations and other sounds have been well studied in the European starling by Jeanne et $\mathrm{al}^{13}$ and Knudsen and Gentner. ${ }^{14}$ Not only are there major differences in firing depending on whether an animal is actively engaged in some learning task, but there is evidence that the selectivity of responses depends on the kinds of sounds that have been previously learned (Figure 9). In particular, neurons in the caudal mesopallium of the forebrain respond with less variability in firing rates to fewer song stimuli during active recognition of biologically meaningful signals compared to passive listening by awake subjects (Figures 5B and 10). Song learning requires auditory feedback to match the vocal output to a song model or a memory trace of such a model. Neurons in the HVC (high vocal center), which is a major 
sensory-motor center, receive input from the caudal mesopallium and other parts of the ascending auditory system and send information or commands to circuits in the song motor pathway (Figure 5B and C). ${ }^{18}$ Recordings of activity in HVC neurons during singing promise to provide new insights about the mechanisms involved in the ontogeny of birdsong. A particularly comprehensive review, which includes important information from comparative studies, is provided by Jarvis. ${ }^{50}$

\section{Coordination of vocalizations and recognition over evolutionary time} Linkage disequilibrium and genetic coupling Mate choice within a population is seldom random. Some individuals (usually males) are preferred as mates because they produce more attractive signals, which may or may not indicate their health or genetic quality. The most widely accepted hypothesis to explain how signal structure and recognition remain coordinated or coupled over evolutionary time is easy to understand: mutual selection by senders and receivers. ${ }^{51}$ If an individual produces signals that diverge too much from the population mean, receivers on average are likely to discriminate against the signaler. If an individual receiver prefers extreme values of signals, it is unlikely to find a mate with an acceptable signal. This mutual selection not only results in relative stability of the communication system but is also reinforced by linkage disequilibrium. That is, if, as usual, males produce mate-attracting signals, their female offspring will inherit the genes responsible for producing signals with attractive properties even though they do not express this phenotype. By the same token, the male offspring of females with a particular preference inherit genes for that preference even though those male offspring do not express this phenotype. Over some generations the genes for vocal structure and preference then become genetically correlated. Paradoxically, as this correlation becomes very strong, female choice of attractive males might become so strong that selection favoring attractive males will result in the rapid evolution of some traits, even to the detriment of male fitness. This scenario is the so-called Fisher effect or runaway sexual selection and may sometimes result in extinction or rapid speciation. ${ }^{51}$

Another idea is that common genes or tightly linked genes might be inherited as a unit and control both the neuromuscular mechanisms underlying signals and the neural mechanisms that recognize these signals. ${ }^{52}$ This idea - termed genetic coupling - was supported by experiments with insects and frogs, showing that females of interspecific hybrids preferred the intermediate signals produced by males of the same cross. ${ }^{1,53}$ Indeed, in two species of crickets Teleogryllus oceanicus and Teleogryllus commodus, there were sex-linked or sex-limited song traits - the songs differed depending on the species of the female - and females of the same cross preferred the songs of their hybrid brothers rather than those of the males of the reciprocal cross. ${ }^{53}$ Female hybrids of crosses between the pine woods tree frog (Hyla femoralis) and Cope's gray tree frog (H. chrysoscelis) also showed behavioral coupling, although there was little indication of any sex-limited expression in hybrids. ${ }^{54} \mathrm{~A}$ simpler explanation is that the preference arises simply because calls and preferences are polygenic traits and hence both are expected to have values intermediate between those of the two parental species. ${ }^{55}$ Genetic analyses of interspecific hybrids and second-generation hybrids, combined with mapping of the genes using quantitative trait loci, have, however, demonstrated that genes for song structure and preferences map to the same loci (or adjacent ones) on the same chromosome in Hawaiian crickets. ${ }^{56}$ This result is predicted by the genetic coupling hypothesis and could not result from simple polygenic inheritance, which would have the expectation that F2 hybrids would show highly variable songs and preferences. Until more robust studies with other species are completed, the prevalence, and hence the evolutionary significance of this mechanism, will remain unknown. The appeal of such a mechanism is that rapid divergent and coordinated evolution could occur because large mutations that would cause substantial changes in signals would simultaneously cause parallel changes in recognition. One neural mechanism proposed in the original formulation of this hypothesis is that both males and females would possess the same neural oscillator, which would be used, for example, to control the temporal patterning of sound pulses by males and as a reference oscillator for the timing of auditory-evoked neural spikes in the female. ${ }^{52} \mathrm{~A}$ mutation would change the timing of such a common oscillator, and both the temporal pattern of the song and the temporal preferences of the receiver would be automatically shifted in unison.

\section{Polyploidy and acoustic communication}

Polyploidy is a process of multiplication of whole sets of chromosomes. Newly formed polyploids are almost always isolated from diploid parental forms because of the chromosome-number difference that renders hybrids inviable or sterile. Polyploid speciation is common in plants, but it has also occurred numerous times in vertebrates. ${ }^{57,58}$ Ohno, ${ }^{57}$ for example, proposes that there were two major polyploidy events in the early history of vertebrates. More recently 
evolved polyploid species - triploids, tetraploids, and even octaploids - are uncommon but widespread among fishes and amphibians. ${ }^{58}$ Among frogs and toads, polyploid species are often cryptic in the sense that they are morphologically similar to the descendants of their diploid parents. Indeed, most polyploid frogs and toads were initially detected by differences in the male advertisement call. ${ }^{58}$

The call differences between diploids and polyploids presently serve to reduce the chances of mismatings between individuals of different ploidy. Two questions thus arise: Does the shift in call properties occur at the time polyploids arise, and is the shift caused by morphological changes associated with polyploidy? Even if polyploids arise spontaneously (autopolyploidy) or after hybridization (allopolyploidy), there are likely to be many fewer individuals compared to the population size of the diploid parents. Polyploids also face the daunting task of competing with the parental forms for resources, and the evolution of different ecological niches that would allow diploids and polyploids to coexist in the same area is likely to require multiple generations of selection. If polyploidization were to immediately foster premating reproductive isolation of newly formed polyploids and their diploid parents, the likelihood of a successful speciation event would be increased.

Japanese scientists studying diploid species provided evidence that changes in call properties occurred in artificially generated polyploids. ${ }^{59}$ In the Japanese tree frog (Hyla japonica), for example, the pulse rate of the advertisement call was about $13 \%$ lower in autotriploids, which were created by coldshocking eggs. Backcrossing autotriploid females to diploid males resulted in some tetraploids, in which the pulse rate was about 20\% lower than that in diploids. Whereas the Japanese tree frog does not have any polyploid relatives, there is evidence that the tetraploid eastern gray tree frog $(H$. versicolor) has independently arisen three or more times from hybridization events involving (ancestral) divergent lineages of the diploid H. chrysoscelis or other extinct closely related species. ${ }^{60,61}$ The nearly identical call structure of males of the three widespread tetraploid lineages, and the fact that female tetraploids of these lineages use the same acoustic criteria, which differ from those of $H$. chrysoscelis (see earlier text), suggests that polyploidy per se may affect both calls and preferences in a parallel fashion. This hypothesis was supported by experiments that showed the following: 1) most male autotriploids of $H$. chrysoscelis shifted the pulse rate of their calls in the direction of the wild-type tetraploids by about $13 \%$, as in the study of the Japanese tree frog; ${ }^{62}$ 2) female autotriploids showed a shift in pulse-rate preference in the same direction and order of magnitude. ${ }^{63}$ Not all of the autotriploid males showed changes in the pulse rate, however. Frogs in which the shift occurred had larger red blood cells - about the same size as those of rare triploid hybrids found in the wild - than did the frogs in which there was no shift. ${ }^{62}$ Cell size in most other tissues, including neurons, is increased in polyploids, ${ }^{64}$ suggesting that the difference in cell size rather than the difference in gene dosage between diploids and autotriploids was responsible for the shift in pulse rate. Studies are currently underway to assess differences in cell size and number in the muscles and auditory neurons that participate in call production and recognition. Because cell size in diploid fish and amphibians is also affected by developmental temperature, cell-size effects on acoustic signals may be widespread in these animals. ${ }^{65}$ If so, this mechanism could constitute an evolutionarily significant genotype-by-environment factor.

\section{Conclusion}

The coordination or coupling of the production of mateattracting sounds and their recognition is crucial to reproductive success. This review considers the mechanisms and evolution of these processes. The focus has been on lower vertebrates, in which unlearned sounds are mainly responsible for mate attraction and recognition, and on songbirds, in which song learning has parallels with human language acquisition. These systems have advantages, in that secondary correlations - temperature effects in lower vertebrates and neuronal activity in birds that are singing or engaged in a learning task - between short-term changes in neural selectivity and behavior boost our confidence that the selectivity for auditory neurons for key properties of mate-attracting signals is not merely coincidental. In terms of evolutionary change, studies of lower vertebrates offer the chance to explore experimentally how genetic coupling and "pleiotropic" effects of speciation by polyploidy can bring about rapid, coordinated changes in both signal structure and recognition.

\section{Disclosure}

The author reports no conflicts of interest in this work.

\section{References}

1. Gerhardt HC, Huber F. Acoustic Communication in Insects and Anurans: Common Problems and Diverse Solutions. Chicago, IL: University of Chicago Press; 2002.

2. Gerhardt HC. Geographic variation in acoustic communication: reproductive character displacement and speciation. Evol Ecol Res. 2013;15:605-632

3. Gerhardt HC. Reproductive character displacement of female mate choice in the grey treefrog Hyla chrysoscelis. Anim Behav. 1994;47:959-969. 
4. Bass AH, McKibben JR. Neural mechanisms and behaviors for acoustic communication in teleost fish. Prog Neurobiol. 2003;69:1-26.

5. Wysocki LE, Ladich F. The representation of conspecific sounds in the auditory brainstem of fishes. J Exp Biol. 2003;206:2229-2240.

6. Gerhardt HC, Schwartz JJ. Auditory tuning and frequency preferences in anurans. In: Ryan MJ, editor. Anuran Communication. Washington, DC: Smithsonian Press; 2001:73-85.

7. Wilczynski W, Keddy-Hector AC, Ryan MJ. Call patterns and basilar papilla tuning in cricket frogs. I. Differences among populations and between sexes. Brain Behav Evol. 1992;39:229-237.

8. Brenowitz EA, Rose G, Capranica RR. Neural correlates of temperature coupling in the vocal communication system of the gray treefrog (Hyla versicolor). Brain Res. 1985;359:364-367.

9. Diekamp BM, Gerhardt HC. Selective phonotaxis to advertisement calls in the gray treefrog Hyla versicolor: behavioral experiments and neurophysiological correlates. J Comp Physiol A. 1995;177:173-190.

10. Edwards CJ, Alder TB, Rose GJ. Pulse rise time but not duty cycle affects the temporal selectivity of neurons in the anuran midbrain that prefer slow AM rates. J.Neurophysiol. 2005;93:1336-1341.

11. Edwards CJ, Leary CJ, Rose GJ. Mechanisms of long-interval selectivity in midbrain auditory neurons: roles of excitation, inhibition, and plasticity. J Neurophysiol. 2008;100:3407-3416.

12. Rose GJ. Time computations in anuran auditory systems. Front Neurobiol. 2014;5:1-7.

13. Jeanne JM, Thompson JV, Sharpee TO, Gentner TQ. Emergence of learned categorical representations within an auditory forebrain circuit. J Neurosci. 2011;31(7):2595-2606.

14. Knudsen DP, Gentner TQ. Active recognition enhances the representation of behaviorally relevant information in single auditory forebrain neurons. J Neurophysiol. 2013;109:1690-1703.

15. Knudsen DP, Gentner TQ. Mechanisms of song perception in oscine birds. Brain Lang. 2010;115:59-68.

16. Meliza CD, Chi Z, Margoliash D. Representations of conspecific song by starling secondary forebrain auditory neurons: toward a hierarchical framework. J Neurophysiol. 2010;103:1195-1208.

17. Schneider DM, Woolley SMN. Discrimination of communication vocalizations by single neurons and groups of neurons in the auditory midbrain. J Neurophysiol. 2010;103:3248-3265.

18. Prather JF. Auditory signal processing in communication: perception and performance of vocal signals. Hearing Res. 2013;305:144-155.

19. Poremba A, Bigelow J, Rossi B. Processing of communication sounds: contributions of learning, memory, and experience. Hearing Res. 2013;305:31-44.

20. Gerhardt HC. Female mate choice in treefrogs: static and dynamic acoustic criteria. Anim Behav. 1991;42:615-635.

21. Gerhardt HC. Evolution of acoustic communication: a multilevel analysis of signal variation. Bioacoustics. 2012;21:9-11.

22. Gerhardt HC. Acoustic spectral preferences in two cryptic species of grey treefrogs: implications for mate choice and sensory mechanisms. Anim Behav. 2005;70:39-49.

23. Ryan MJ, Perrill SA, Wilczynski W. Auditory tuning and call frequency predict population-based preferences in the cricket frog, Acris crepitans. Am Nat. 1992;139:1370-1383.

24. Gerhardt HC, Dyson ML, Tanner SD. Dynamic properties of the advertisement calls of gray treefrogs: patterns of variability and female choice. Behav Ecol. 1996;7:7-18.

25. Wells KD, Taigen TL. The effect of social interactions on calling energetics in the gray treefrog (Hyla versicolor). Behav Ecol Sociobiol. 1986;19:9-18.

26. Welch AM, Smith MJ, Gerhardt HC. A multivariate analysis of genetic variation in the advertisement call of the gray treefrog Hyla versicolor. Evolution. 2014;68(8):1629-1639.

27. Welch AM, Semlitsch RL, Gerhardt HC. Call duration as an indicator of genetic quality in male gray treefrogs. Science. 1999;280: 1928-1930.
28. Gerhardt HC, Brooks R. Experimental evidence of multivariate female choice in gray treefrogs (Hyla versicolor): evidence for directional and stabilizing selection. Evolution. 2009;63:2504-2512.

29. Kroodsma D. The diversity and plasticity of birdsong. In: Marler P, Slabbekoorn H, editors. Nature's Music: The Science of Birdsong. San Diego, CA: Elsevier Academic Press; 2004:108-131.

30. Doherty JA, Gerhardt HC. Evolutionary and neurobiological implications of selective phonotaxis in the spring peeper (Hyla crucifer). Anim Behav. 1984;32:875-881.

31. Gerhardt HC. Acoustic communication in two groups of closely related treefrogs. Adv Stud Behav. 2001;30:99-167.

32. Gerhardt HC. Advertisement-call preferences in diploid-tetraploid treefrogs (Hyla chrysoscelis and Hyla versicolor): implications for mate choice and the evolution of communication systems. Evolution. 2005;59: 395-408.

33. Narins PM, Feng AS, Fay RR, Popper AN, editors. Hearing and Sound Communication in Amphibians. New York, NY: Springer Science + Business Media; 2007.

34. Plakke B, Diltz MD, Romanski LM. Coding of vocalizations by single neurons in ventrolateral prefrontal cortex. Hearing Res. 2013;305: 135-143.

35. Gerhardt HC, Klump GM. Masking of acoustic signals by the chorus background noise in the green treefrog: a limitation on mate choice. Anim Behav. 1988;36:1247-1249.

36. Bee MA. Sound source segregation in grey treefrogs: spatial release from masking by the sound of a chorus. Anim Behav. 2007;74:549-558.

37. Bee MA, Micheyl C. The cocktail party problem: What is it? How can it be solved? And why should animal behaviorists study it? J Comp Psychol. 1988;122:235-251.

38. Bee MA. Treefrogs as animal models for research on auditory scene analysis and the cocktail party problem. Int J Psychophysiol. 2015. In press.

39. Feng AS, Narins $\mathrm{PM}, \mathrm{Xu} \mathrm{CH}$, et al. Ultrasonic communication in frogs. Nature. 2006;440:333-336.

40. Gerhardt HC, Doherty JA. Acoustic communication in the gray treefrog, Hyla versicolor: evolutionary and neurobiological implications. J Comp Physiol A. 1988;162:261-278.

41. Gerhardt HC, Schul J. A quantitative analysis of behavioral selectivity for pulse rise-time in the gray treefrog, Hyla versicolor. J Comp Physiol A. 1999; 185:33-40.

42. Schul J, Bush SL. Non-parallel coevolution of sender and receiver in the acoustic communication system of treefrogs. Proc R Soc Lond B. 2002;269:1847-1852.

43. Gayou DC. Effects of temperature on the mating call of Hyla versicolor. Copeia. 1984:733-738.

44. Gerhardt HC. Temperature coupling in the vocal communication system of the gray treefrog Hyla versicolor. Science. 1978;99:992-994.

45. Brantley RK, Bass AH. Alternative male spawning tactics and acoustic signals in the plainfin midshipman fish, Porichthys notatus (Teleostei, Batrachoididae). Ethology. 1994;96:213-232.

46. Gerhardt HC. Mating call recognition in the green treefrog (Hyla cinerea): importance of two frequency bands as a function of sound pressure level. J Comp Physiol. 1981;144:9-16.

47. Gerhardt HC, Mudry KM. Temperature effects on frequency preferences and mating call frequencies in the green treefrog, Hyla cinerea (Anura: Hylidae). J Comp Physiol. 1980;137:1-6.

48. Simmons DD, Meenderink SWF, Vassilakis PN. Anatomy, physiology, and function of auditory end-organs in the frog inner ear. In: Narins PM, Feng AS, Fay RR, Popper, AN, editors. Hearing and Sound Communication in Amphibians. New York, NY: Springer Science + Business Media; 2007:184-220.

49. Endepols H, Feng AS, Gerhardt HC, Schul J. Walkowiak W. Roles of the auditory midbrain and thalamus in selective phonotaxis in female gray treefrogs (Hyla versicolor). Behav Brain Res. 2003;145: 53-77. 
50. Jarvis ED. Brains and birdsong. In: Marler P, Slabbekoorn H, editors. Nature's Music: The Science of Birdsong. San Diego, CA: Elsevier Academic Press; 2004:226-271.

51. Andersson, M. Sexual Selection. Princeton, NJ: Princeton University Press; 1994.

52. Alexander RD. Evolutionary change in cricket acoustic communication. Evolution. 1962;16:443-467.

53. Hoy RR, Paul RC. Genetic control of song specificity in crickets. Science. 1973;180:82-83.

54. Doherty JA, Gerhardt HC. Hybrid treefrogs: vocalizations of males and selective phonotaxis of females. Science. 1983;220:82-113.

55. Butlin RK, Ritchie MG. Genetic coupling in mate recognition systems: What is the evidence? Biol J Linnean Soc. 1989;37:237-246.

56. Shaw KL, Lesnick SC. Genomic linkage of mate song and female acoustic preference QTL underlying a rapid species radiation. Proc Natl Acad Sci U S A. 2009;106:9737-9742.

57. Ohno S. Evolution by Gene Duplication. Heidelberg, Germany: Springer; 1970.

58. Mable BK, Alexandrou MA, Taylor MI. Genome duplication in amphibians and fish: an extended synthesis. J Zool. 2011;284:151-182.

59. Ueda H. Mating calls of autotriploid and autotetraploid males in Hyla japonica. Sci Rep Lab Amphib Biol Hiroshima Univ. 1993;12:177-189.

60. Ptacek MB, Gerhardt HC, Sage RD. Speciation by polyploidy in treefrogs: multiple origins of the tetraploid, Hyla versicolor. Evolution. 1994;48:898-908.
61. Holloway AK, Cannatella DC, Gerhardt HC, Hillis DM. Polyploids with different origins and ancestors form a single polyploidy species. Am Nat. 2006;167:E88-E101.

62. Keller MD, Gerhardt HC. Polyploidy affects call structure in gray treefrogs. Proc R Soc Lond B. 2001;268:341-345.

63. Tucker MA, Gerhardt HC. Parallel changes in mate-attracting calls and female preferences in autotriploid tree frogs. Proc $R$ Soc Lond B. 2011;279:1583-1587.

64. Szaro BG, Tompkins R. Effect of tetraploidy on dendritic branching in neurons and glial cells of the frog, Xenopus laevis. J Comp Neurol. 1987;258;304-316.

65. Arendt JD. The cellular basis for phenotypic plasticity in body size in western spadefood toad (Spea hammondi) tadpoles: patterns of cell growth and recruitment in response to food and temperature manipulations. Biol J Linn Soc Lond. 2006;88:499-510.

66. Squire LR. Encyclopedia of Neuroscience. 3rd ed. Adelman et al, editors. New York, NY: Oxford University Press; 2004.

67. Gerhardt, HC. Acoustic properties used in call recognition by frogs and toads. In Fritzsch B, Ryan MJ, Wilczynski W, Hetherington TE Walkowiak W eds. The Evolution of the Amphibian Auditory System John Wiley and Sons, NY, USA; 1988:455-483.
Open Access Animal Physiology

\section{Publish your work in this journal}

Open Access Animal Physiology is an international, peer-reviewed, open access journal publishing original research, reports, reviews and commentaries on all areas of animal physiology. The manuscript management system is completely online and includes a very

\section{Dovepress}

quick and fair peer-review system. Visit http://www.dovepress.com/ testimonials.php to read real quotes from published authors. 